\title{
Weaving Thoughts and Reality through Drawing: New Technologies and Emerging Cognitive and Epistemological Paradigms
}

\author{
Lorenzo Ceccon
}

Virginia Vecchi

\section{Abstract}

The power of drawing as an effective tool for communicating ideas and to represent reality is a well-known topic. However, drawing has shown a much greater potential for knowledge creation, as it can be purposefully used to link and connect ideas and aspects taken from reality, and according to some Scholars it is at the core of the scientific revolution itself. Recent advancements in digital technologies are further increasing the relevance of drawing as a means to create knowledge, especially as regards the purposeful interplay of humans and machines in devising hypotheses and in testing them across disciplines and data types. Are we at the surge of a new scientific paradigm?

Keywords

drawing, knowledge, graphs, Al, digital twin, epistemology.

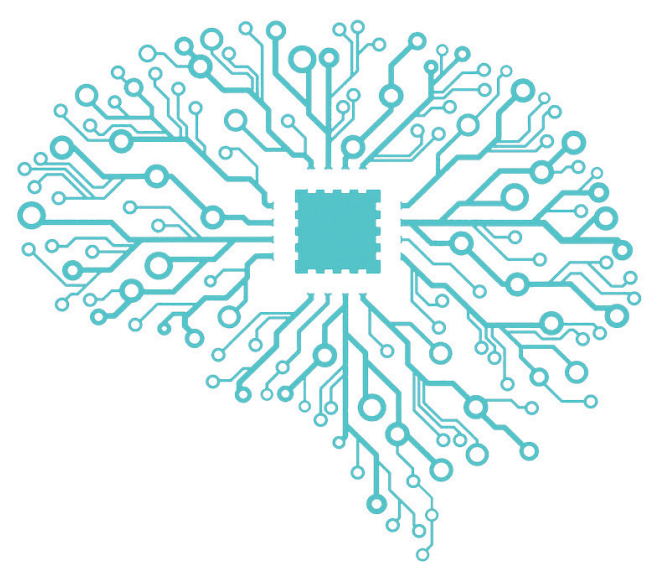


Even without referring to Chinese logograms and in general to pictograms along human history, nor to the pictorial origins of our European alphabets, it is everyday experience that drawings are globally used for expressing concepts when we want to reach quickly the widest possible audience beyond language barriers: road signs, instruction leaflets and alike are a clear example thereof.

If we go beyond such rather banal observation, we can widen the discourse about drawing as an overall universal language category. In fact, from the simplest mental maps to the most advanced forms of digital drawing techniques we will analyse hereinafter, drawing seems a necessary language to not only express, but also to structure thoughts in the first place. It has the power of cancelling distances, not only among people and places -allowing for cross-cultural and global communication- but also among thoughts and knowledge domains, allowing for knowledge contamination and accrual, which is in turn a powerful basis for creativity and new knowledge.

In this paper we will analyse the power of drawing, especially as regards new digital tools, in three steps. First, as a way of structuring thoughts and knowledge. Then, as a way to create a two-way connection to reality. Finally, as a fundamental middle pivotal point linking thoughts and reality to better grasp its most relevant features and to effectively act on it.

\section{Drawing/Thoughts}

It has been argued that "no scientific discipline exists without first inventing a visual and written language" [Latour 1986, p. 13]. For instance, "Chemistry becomes powerful only when a visual vocabulary is invented that replaces the manipulations by calculation of formulas. Chemical structure can be drawn, composed, broken apart on paper, like music or arithmetic, all the way to Mendeleiev's table" [Latour 1986, p. 14].

The importance of drawing as the backbone of structured thinking - both at the personal level of mental maps and as a way to effectively communicate those maps - has been gaining momentum over the last years, also based on evidence showing that it corresponds to an enhanced cognitive performance. Indeed, comparing "instructional materials enhanced with textual metaphors versus graphical metaphors [...] graphical metaphors improve participants' performance" [Mckay 1999, p. 47I]. Another recent study [Barmpoutis 2019] has shown that an emoticon-like scripting language has significantly improved students' performance compared to a purely text-based scripting language.

Moreover, the parallel advancements in the fields of Al and psychology are generating cross-fertilization and spill-overs, whereby the visuo-spatial reasoning -hence the drawing substrate of thinking - lies at the core of such parallelism. "One path for joining psychology and $\mathrm{Al}$ is to evaluate computational models in $\mathrm{Al}$ as helpful frameworks for constructing computational models in psychology. [...] Building computational models of visuospatial reasoning should help computer scientists create effective Al programs, cognitive scientists understand human reasoning, and educators design effective instruction" [Reed 2020, p. 820]. In fact, "At present, contemporary Al and the mind and brain sciences are converging in an increasing number of areas as machine learning techniques are encroaching into the modelling toolkits of researchers in the mind and brain sciences, and neuroscience is increasingly being used to validate Al Results" [Chaudhary 2020, p. 367].

Fig. I. Map of Königsberg in Euler's time showing the actual layout of the
seven bridges, highlighting the river Pregel and the bridges, reaching then
the Euler's graph. [CC BY-SA 3.0].

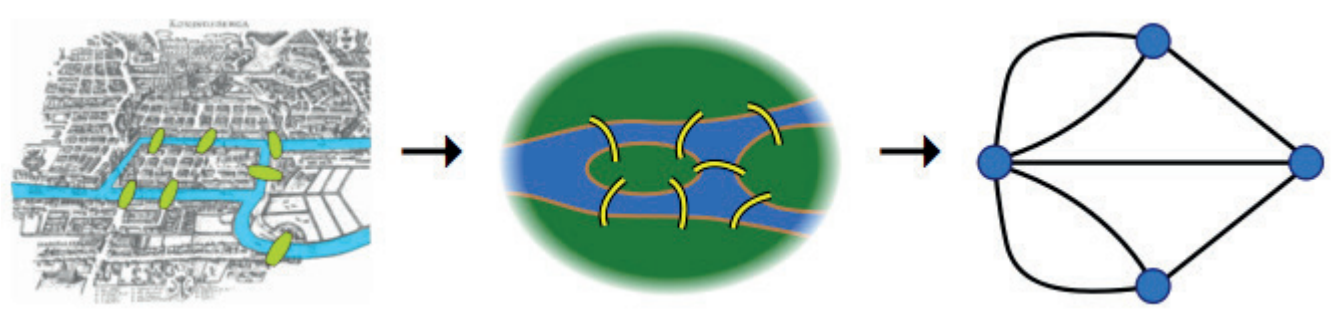


Fig. 2. Comparison of the graphs of the Seven bridges of Konigsberg (top) and Five room puzzle (bottom). The numbers denote the number of edges connected to each node. Nodes with an odd number of edges are shaded orange. [Cmglee personal work on Norris F. R. 1985, CC BY-SA 3.0]

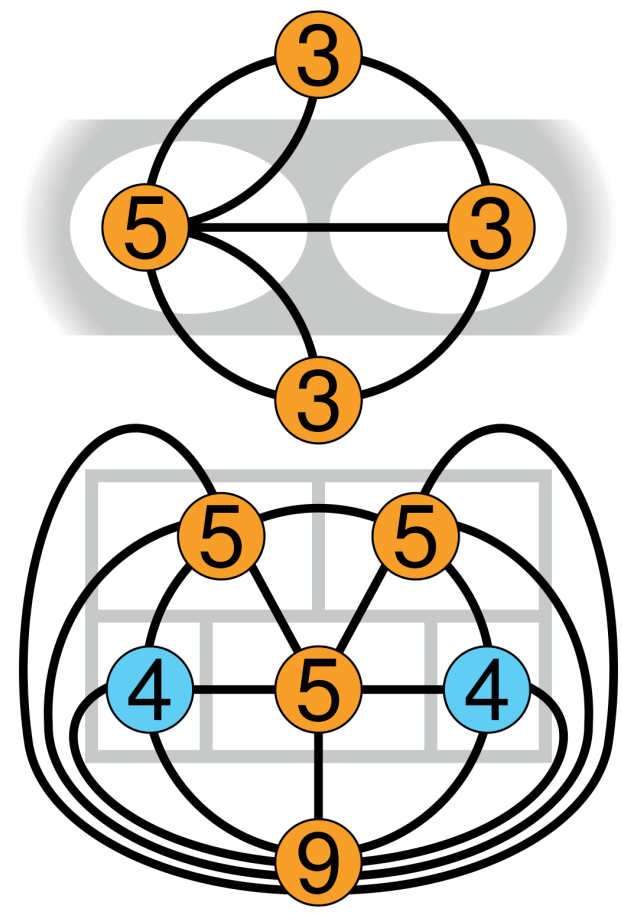

While the use of a series of different kinds of drawing categories, ranging from the use of symbols to representation techniques such as perspective, can all help visualize and clarify thoughts, and even simulate imagined realities, a very interesting subset of drawing techniques relating to thought structuring lies in graphs. The use of graphs has been fundamental in tackling a series of scientific topics, as in the famous Königsberg's bridges problem, and newer techniques based thereupon including mind mapping tools and Al techniques have been adopted in many disciplines, such as business strategy, in order to (self-)brainstorm, analyse and communicate complex ideas about complex systems.

In fact, what are the main aspects of drawing underlying such convergence of the human and the artificial mind? Some Scholars have put forward a "proposal of many hundreds of high-dimensional internal cognitive maps through which our perceptions unfold" [Churchland 2012, p. I]. "Hence, scientific research and theorizing are reconceived of as modifying and amplifying conceptual maps in the neuronal activation space, which, it is believed, may be simulated in computer hardware without the constraints of human neurobiology" [Chaudhary 2020, p. 368]. In other words, drawing -and in particular the Knowledge Graphs underlying some cutting-edge Al systems- can be seen as the most effective way of mapping our own human internal cognitive maps and taking them to the next level.

Al based on Graph Neural Networks has been deployed to not only statically structure a system of pre-defined thoughts. On the contrary, it has been found effective in the creation of an iterative process "assisting in forming causal and conceptual models. In order to utilize the insights gained in this procedure, a crucial step is to formalize, develop \& test techniques to channel human-originated feedback back into the automated decision-making process" [Holzinger 2021, p.34]. Indeed, "In many fields the aim is to build systems capable of explaining themselves, engaging in interactive what-if questions. [...] Graph Neural Networks play a major role for multi-modal causability, since causal links between features can be defined directly using graph structures" [Holzinger 202I, p. I].The use of Knowledge Graphs is not only a very effective tool to ground $\mathrm{Al}$ and Al-assisted conceptual modelling; it is also a major step towards the interpretability of Al "thinking", (partially) overcoming one of the main criticisms about Al, the black-box effect. "The intrinsic characteristics of graph models make them suitable for the analysis of complex systems, while still providing highly interpretable results" [Barbiero 2020, p. 2]. 
Fig. 3. Graph fusion. Data from four different input modalities time-series, images, knowledge databases and text- mapped into an interaction \& correspondence graph (ICG) can generate positive/ egative samples akin o word-cooccurrences. [Holzinger 2021, p.30].

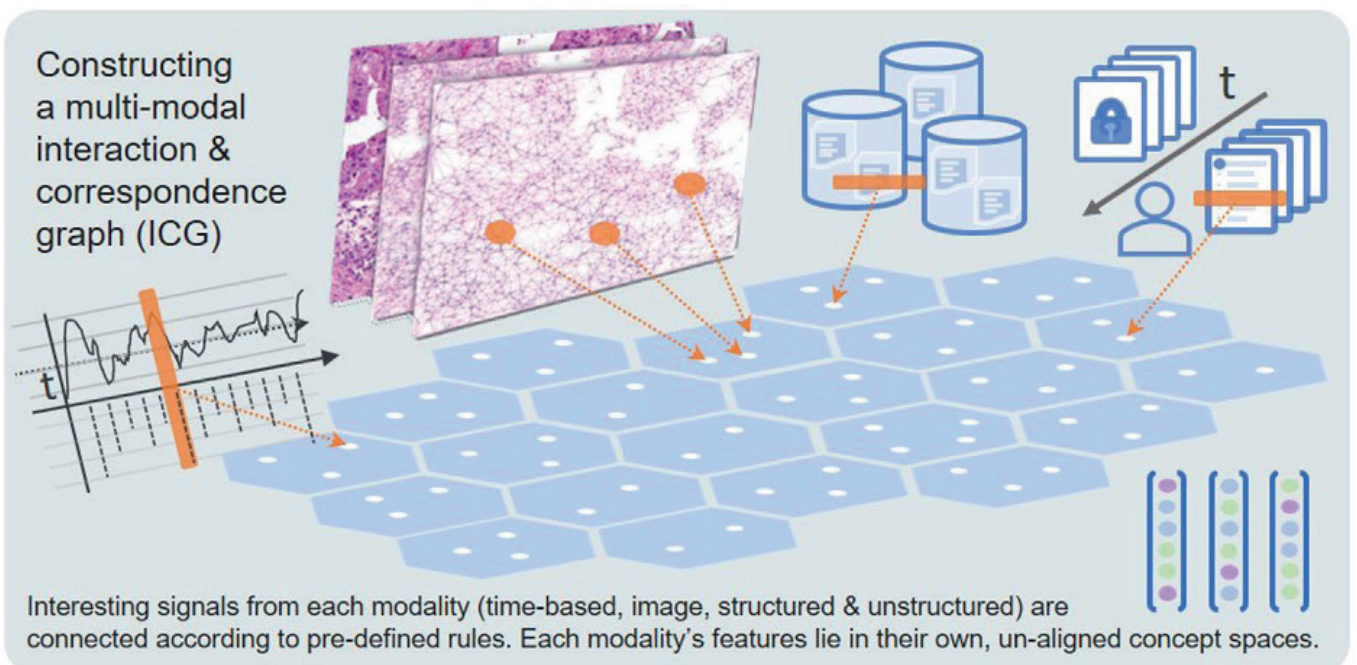

\section{Drawing/Reality}

Drawing can be utilized to not only effectively represent thoughts and their connections among themselves, but also as a way to represent reality in a biunivocal relationship. "The rationalization that took place during the so-called "scientific revolution" is not of the mind, of the eye, of philosophy, but of the sight [...] since the picture moves without distortion it is possible to establish, in the linear perspective framework, what he calls a 'two way' relationship between object and figure" [Latour 1986, p. 7].

A first important aspect of the two-way relationship between drawing and reality is the concept of "ontologies", i.e. a categorization of (objects from) reality following a semantic logic. A categorization based on ontologies is at the core of Knowledge Graphs, which are hence a powerful tool not only to structure thoughts, but also to represent reality in a semantically structured way. "The knowledge representation experts who specialize in semantics-driven ontologies will make no bones about it: a knowledge graph is necessarily built on semantics. Semantics, they argue, is the basis for creating new inferences from the data
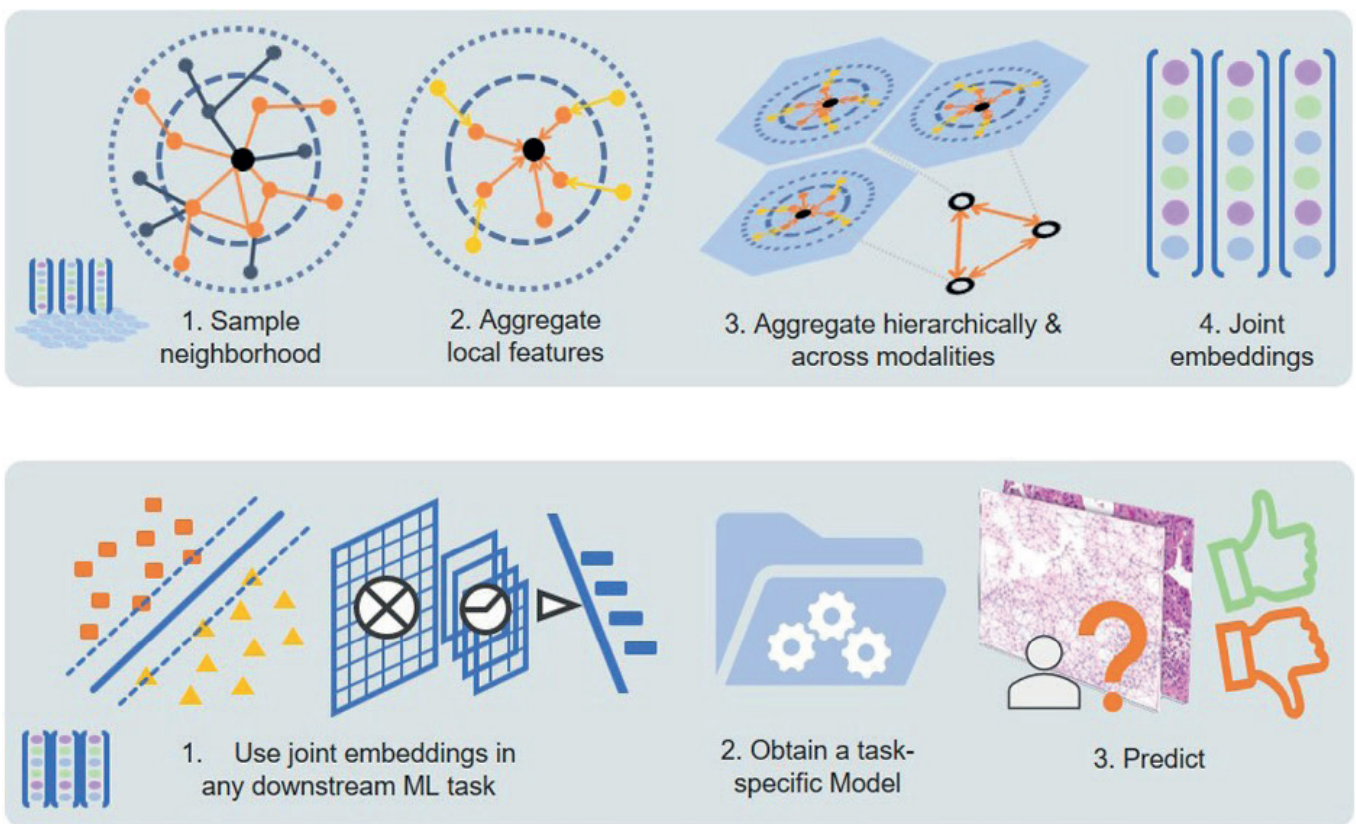

Fig. 4, 5. "Learning hierarchies \& logical graph" [Holzinger 202 I, p.32] 
Fig. 6. Virtual Model of the new Campus of Politecnico di Milano before construction in 2018 [by the Author]. The model is now being used as a base for simulatin spatial hypothesis as to the fruition by different users categories, and as a basis for an lo Digital Twin. which would otherwise go unseen. It's the difference between something that generates new knowledge and a database laying dormant, waiting to be queried. Anything less is just a labelled graph" [Spencer 2018].

Another insightful link between drawing and reality which have emerged following the rise of digital technologies is constituted by Virtual Reality and Digital Twin models. Virtual Reality relies on drawing tools simulating reality based on physical and rendering engines which respectively compute and apply the laws of physics and the laws of perspective, thus achieving a realistic simulation. Yet, another key step allowed by most recent converging technologies - such as loT sensors combined with Al and with physical and rendering engines, among others - is the possibility to "close the simulation circle' between reality and the model: similarly to the well-known scientific paradigm advocated by Karl Popper [Popper 1934], the model is not a one-shot hypothesis on how reality works, but it is an ongoing process wherein feedback from reality helps reshape and adjust it, so that it can run in parallel to reality. This is the so-called Digital Twin. It may refer not only to models simulating the physical-geometrical aspects of reality, but also to its more abstract structure, as for instance that contained in Knowledge Graphs extrapolated therefrom. In a recent piece of research about the use of graph models applied to healthcare, "the graph model represents a virtual prototype of patients, a sort of "digital twin" mirroring the actual multiscale biological system, thus providing a general and flexible framework to run probabilistic simulations" [Barbiero 2020, p. 2].

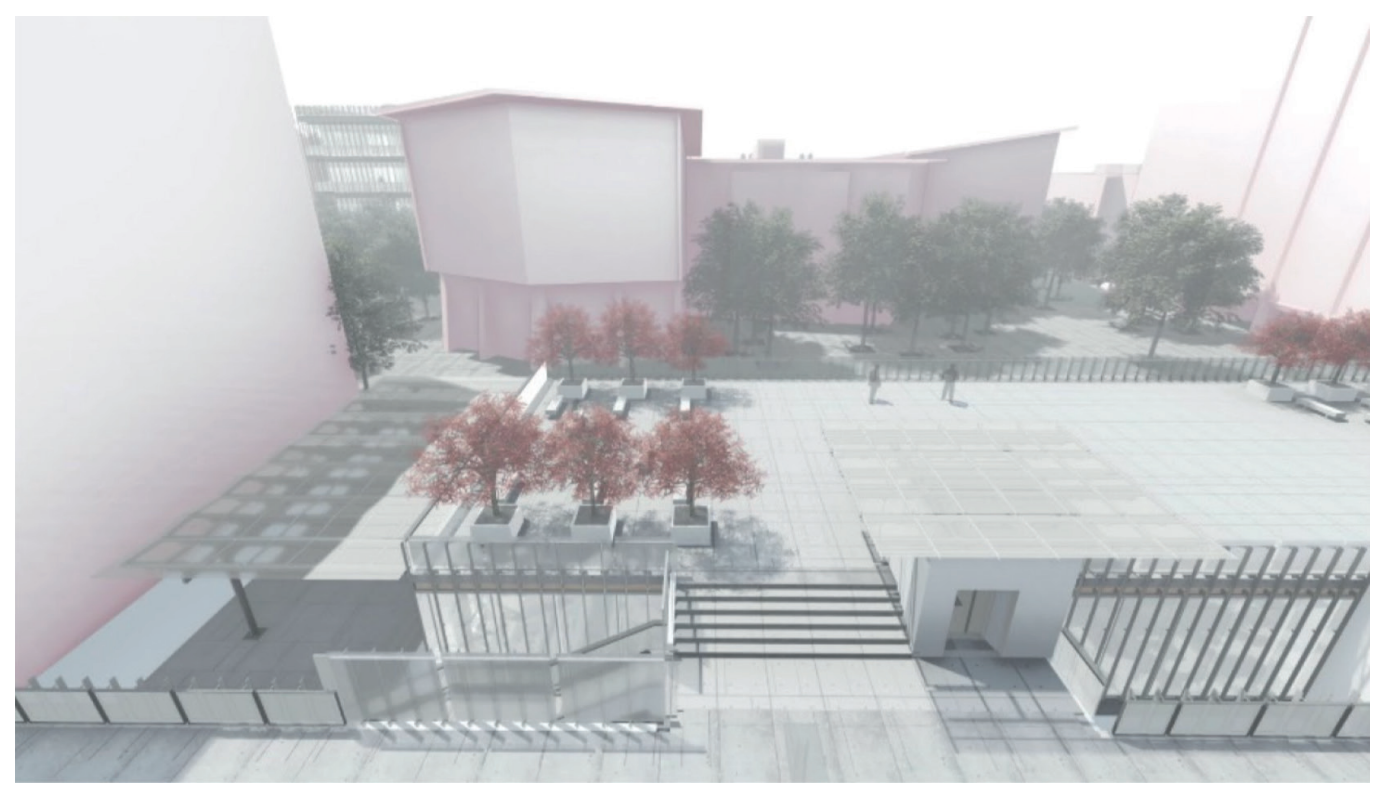

\section{Thought/Drawing/Reality}

After analysing the relationship between drawing and thoughts, and between drawing and reality, we will now propose a combined, organic perspective on how drawing can constitute the main medium bounding together thoughts and reality. The relevance of the link between tough and reality through drawing has been highlighted by Latour who noticed that "By working on papers alone, on fragile inscriptions which are immensely less than the things from which they are extracted, it is still possible to dominate all things, and all people" [Latour 1986, p. 30].

In particular, after establishing the close, two-way bonds drawing/thought and drawing/ reality, we can now consider their interplay. On the one hand, drawing can represent a 
hypothetical reality, a pure projection of mental worlds. In fact, "Perspective is not interesting because it provides realistic pictures; on the other hand, it is interesting because it creates complete hybrids" [Latour 1986, p. 8]. On the other hand, the availability of data from reality, which are embedded in reality simulations - such as a Digital Twin and Knowledge Graphs- can provide a sound knowledge base not only to mentally grasp reality, but also to test hypotheses about how reality is or might be transformed. Digital Twins can in fact be used as a sort of proxy of reality for testing the cognitive hypotheses formulated by humans, since they contain the explicit or implicit knowledge developed by maintaining them aligned to reality along time, and are supposed to have learned to evolve in line with how reality does or would.

Recently, Al techniques and 'Knowledge Graphs' have been used in connection to floor plan drawings to unveil hidden relationships among spaces and their configurations. "The design of floorplans can leverage machine intuition to generate and qualify potential design options. [...] we address a specific abstraction of space: adjacency. Any floorplan carries its own embedded logic, in clear, the relative placement of rooms and their connections is driven by a certain logic of interdependence, and yields varying qualities across space. [...] We later turn to Bayesian modelling to generate adjacency graphs, either freely or under set constraints. By qualifying and generating, our hope is to investigate both sides of the same problem: the understanding of relationships among neighbouring spaces" [Chaillou 2020, p. 2]. In such cases, we see drawings (the graphs) used in connection to other drawings (floorplans), in order to create new floorplans -hence new drawings- which in turn are aimed at designing real world spaces.

The interplay between mind and simulated reality can also operate conversely: the usefulness of a 'human-in-the-loop' as regards Digital Twin models based on visual Al has been shown to increase the efficacy and the accuracy of the simulations, and of the decision making based thereupon. "Due to the fact that humans are unable to directly orient themselves in high dimensional data sets, we need to design, develop and evaluate subspace visualization methods $[84,85]$ to let the human expert interactively manipulate automatically generated samples, thereby iteratively assisting in forming causal and conceptual models. In order to utilize the insights gained in this procedure, a crucial step is to formalize, develop \& test techniques to channel human-originated feedback back into the automated decision-making process" [Holzinger 2021, p. 34].

As a result of the foregoing intertwining of thoughts, drawings and reality, "This new informational environment subsumes both cyber and physical space into a unified artificially constructed virtual world, which is being transposed over the physical world. This superimposed simulated model of the world is where the activities of artificial agents in various embodiments occur. [...] This leads us to the third reconfiguration, which is the elimination of the dualistic divide between humans and the physical world through the monism of an informational worldview, which entails ontological continuity between humans and artificial agents in a new informational environment" [Chaudhary 2020, pp. 375, 376].

Fig. 7. "Generating a counterfactual graph (CG) by sampling from a trained model. A humanin-the-loop interacts with and refines the CG which is subsequently reduced is subsequently reduced to an easily-interpretable decision tree translated back to the oniginal muti[Holzinger 202 I, p. 34]

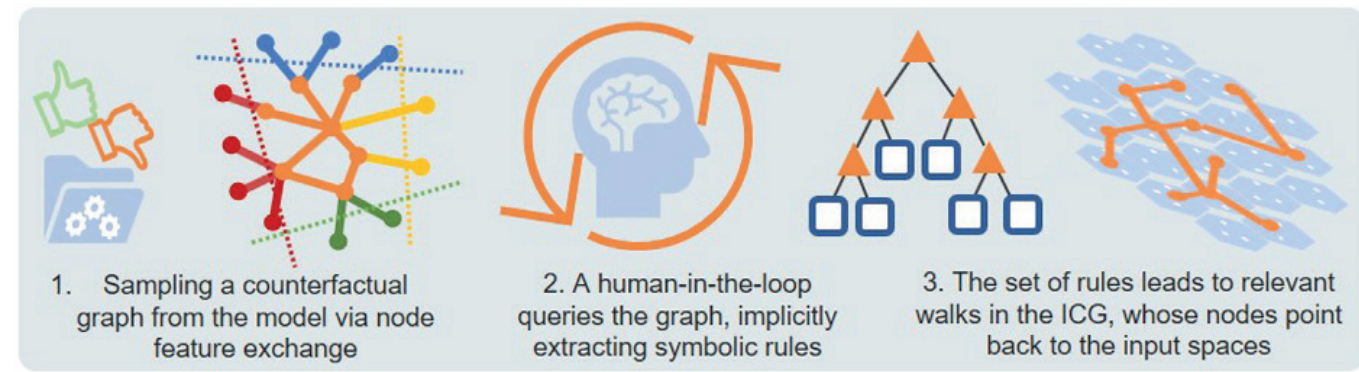




\section{Conclusion}

To sum up, drawing -in all its wide-ranging declinations- seems not a tool of the past but is actually gaining and even increased momentum in times of digital revolution. Drawing techniques are helpful to help support our own visual-spatial reasoning, as well as Al based algorithms, making them at the same time more interpretable and more controllable. At the same time, drawing seems now able to capture reality not only in its more traditional geometrical features, but to even incorporate semantic aspects thereof, as well as a series of explicit or implicit data and knowledge-bases stemming from cutting edge IT technologies, leading to (drawn) models running parallel to reality. We then tried to highlight the potential lying in the interplay between such features as a means to increase knowledge about reality, both by testing hypotheses within simulated environments and by interacting with $\mathrm{Al}$ tools in the endeavour of making sense of a growing number of data and data categories taken from reality. Hence, the potential impact on the very same epistemological paradigms of knowledge creation cannot be overestimated and will require careful scrutiny over the next years.

\section{References}

Baldin S. (2015). The Fuzzy Logic and the Fuzzy Approach:A Comparative Law Perspective. In Poliarchie/Polyarchies, vol.5, p. 22 Trieste: EUT Edizioni Università di Trieste <http://hdl.handle.net/ I0077// I 842> (accessed 2021, 5 February).

Bandemer H., Gebhardt A. (2000). Bayesian fuzzy kriging. In Fuzzy Sets and Systems, n. I I 2, pp. 405-4I 8.

Barbiero P. et al. (2020). Graph representation forecasting of patient's medical conditions: towards a digital twin. [Preprint] arXiv:2009.08299vl [stat.ML] I 7 Sep 2020.

Barmpoutis A., Huynh K. (2019). Name Tags and Pipes: Assessing the Role of Metaphors in Students' Early Exposure to Computer Programming Using Emoticoding. In Nazir S. Teperi AM. Polak-Sopińska A. (Eds.). Advances in Human Factors in Training, Education, and Learning Sciences. AHFE 20 I8. Advances in Intelligent Systems and Computing, vol. 785. Cham: Springer.

Bijanzadeh E., Marzieh M. (20I3). The use of fuzzy- AHP methods to assess fertility classes for wheat and its relationship with soil salinity: east of Shiraz, Iran: A case study. In AJCS. n.7(I I), pp. I 699- I 706.

Chaillou S. (2020). Architecture as a Graph. In Towards data Science. <https://towardsdatascience.com/architecture-as-a-graph-6a835d46f9 I8> (accessed 2021, May 22).

Chaudhary M.Y. (2020). The Artificialization of Mind and World in Artificial Intelligence and Robotics: Contributions from the Science and Religion Forum with Gillian K. Straine, Zygon, In Journal of Religion and Scienze, vol. 55, n. 2, pp.36I-38I.

Chen T.J., Krishnamurthy V.R. (2020). Investigating a Mixed-Initiative Workflow for Digital Mind- Mapping, In Journal of Mechanical Design. vol. 142, pp. $101-404$

Chien L., Buehrer D. (2008). A Visual Lambda-Calculator Using Typed Mind-Maps. In Computer and Electrical Engineering, International Conference on. pp. 250-255.

Čyras V., Lachmayer F. Lapin K. (20 I5). Structural Legal Visualization. In Informatica, vol. 26, n. 2, pp. $199-219$.

Da Costa Pereira C. (2017). Combining Fuzzy Logic and Formal Argumentation for Legal Interpretation. In Proceedings of the I 6th Edition of the International Conference on Artificial Intelligence and Law. London, United Kingdom 20 I7. pp.49-58. New York: Association for Computing Machinery.

Euler L. ( I 736). Solutio problematis ad geometriam situs pertinentis. In Comment. Acad. Sci. U. Petrop, n. 8, pp. I 28-40. Ed. Nova.

Gudwin R., Queiroz J. (2005). Towards an introduction to computational semiotics. In International Conference on Integration of Knowledge Intensive Multi-Agent Systems. I 8-2 I April 2005, pp. 393-398. Waltham, MA IEEE.

Holzinger A. et al. (202I). Towards multi-modal causability with Graph Neural Networks information fusion for explainable Al. In Information Fusion. n. 71, pp. 28-37.

Koyama Y. (20 I 6). Computational Design Driven by Visual Aesthetic Preference, A DoctorThesis Degree of Doctor of Information Science and Technology in Computer Science. Thesis Supervisor Igarashi T. University of Tokyo.

Latour B. (1986), Visualisation and Cognition: Drawing Things Together. In H. Kuklick (Ed.). Knowledge and Society Studies in the Sociology of Culture Past and Present, vol. 6, pp. I-40. Stamford: Jai Press.

Legrand J. (1999), Some guidelines for fuzzy sets application in legal reasoning. In Artificial Intelligence and Law, n. 7, p. 235.

Mckay E. (1999). Exploring the Effect of Graphical Metaphors on the Performance of Learning Computer Programming Concepts in Adult Learners: a pilot study. In Educational Psychology. n. 19 (4), pp.47 I-487. 
Mokarram M., Najafi-Ghiri M. (20 I6). Combination of Fuzzy Logic and Analytical Hierarchy Process Techniques to Assess Potassium Saturation Percentage of Some Calcareous Soils (Case Study: Fars Province, Southern Iran). In Agriculture. n 6(4), p. 59.

Norris S. (2018).Where Ontologies End and Knowledge Graphs Begin. In ODSC. <https://opendatascience.com/where-ontologies-end-and-knowledge-graphs-begin/> (accessed 202I, 5 February).

Ovsjanikov M. (2017). Functional View of Geometry Processing: Operator-based Techniques for Shape Analysis. Computer Vision and Pattern Recognition [cs.CV].In HAL. Université Paris-Sud Orsay <https://hal.archives-ouvertes.fr/tel-0I55559|> (accessed 2021, 5 February).

Popper K. (1934). Logik der Forschung. Vienna: Springer.

Reed S. K. (2020). Searching for the Big Pictures. In Perspectives on Psychological Science, 2020, vol. I 5(3), pp. 8 I 7-830.

Runberger J. Lienhard J. (2018). Collaborative Models for Design Computation and Form Finding -NewWorkflows in Versioning Design Processes. In De Rycke K. et al. (Eds.). Humanizing Digital Reality, pp. 463-478. Singapore: Springer.

Sherwin R. K. (200I).Visualizing Law in the Age of the Digital Baroque: Arabesques \& Entanglements. In Books, n. 3. Routledge <https://digitalcommons.nyls.edu/fac_books/3> (accessed 202I, 5 February).

Shields R. (2012). Cultural Topology:The Seven Bridges of Königsburg I736. In Theory, Culture \& Society, n. 29 (4-5), pp. $43-57$.

\section{Authors}

Lorenzo Ceccon, Politecnico di Milano, lorenzo.ceccon@polimi.it Virginia Vecchi, Politecnico di Milano, virginia.vecchi@polimi.it

To cite this chapter. Ceccon Lorenzo, Vecchi Virginia (2021). Weaving Thoughts and Reality through Drawing: New Technologies and Emerging Cognitive and Epistemological Paradigms. In Arena A., Arena M., Mediati D., Raffa P. (a cura di). Connettere. Un disegno per annodare e tessere. Linguaggi Distanze Tecnologie. Atti del $42^{\circ}$ Convegno Internazionale dei Docenti delle Discipline della Rappresentazione/Connecting. Drawing for weaving relationship. Languages Distances Technologies. Proceedings of the 42th International Conference of Representation Disciplines Teachers. Milano: FrancoAngeli, pp. $2173-2180$. 